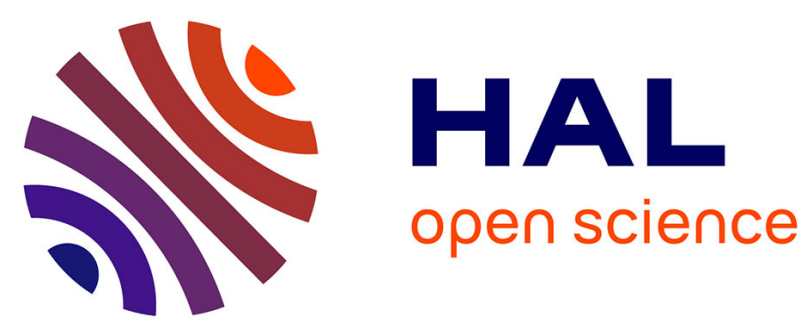

\title{
3D unsteady computations of evaporative instabilities in a sessile drop of ethanol on a heated substrate
}

\author{
Sergey Semenov, Florian Carle, Marc Medale, David Brutin
}

\section{To cite this version:}

Sergey Semenov, Florian Carle, Marc Medale, David Brutin. 3D unsteady computations of evaporative instabilities in a sessile drop of ethanol on a heated substrate. Applied Physics Letters, 2017, 111 (24), pp.241602. 10.1063/1.5006707 . hal-01769992

\section{HAL Id: hal-01769992 \\ https://hal.science/hal-01769992}

Submitted on 19 Apr 2018

HAL is a multi-disciplinary open access archive for the deposit and dissemination of scientific research documents, whether they are published or not. The documents may come from teaching and research institutions in France or abroad, or from public or private research centers.
L'archive ouverte pluridisciplinaire HAL, est destinée au dépôt et à la diffusion de documents scientifiques de niveau recherche, publiés ou non, émanant des établissements d'enseignement et de recherche français ou étrangers, des laboratoires publics ou privés. 


\title{
3D unsteady computations of evaporative instabilities in a sessile drop of ethanol on a heated substrate
}

\author{
Sergey Semenov, ${ }^{1,2}$ Florian Carle, ${ }^{1,3}$ Marc Medale, ${ }^{1}$ and David Brutin ${ }^{1,4, *}$ \\ ${ }^{1}$ Aix-Marseille University, IUSTI UMR 7343 CNRS, 13453 Marseille, France \\ ${ }^{2}$ Aix-Marseille University, MADIREL UMR 7246 CNRS, 13013 Marseille, France \\ ${ }^{3}$ Department of Mechanical Engineering and Materials Science, \\ Yale University, New Haven, Connecticut 06511, USA \\ ${ }^{4}$ Institut Universitaire de France, 75231 Paris, France
}

(Dated: April 19, 2018)

\begin{abstract}
Droplets are ubiquitous and have been studied for century. However, the flow pattern and instabilities occurring during evaporation are still under investigations and their origin is still debated. In this letter, we are comparing an ethanol drop evaporating onto a heated substrate under weightlessness conditions and with pinned contact line with a 3D unsteady computation of thermo-convective instabilities to determine with accuracy the type of instabilities. Our one-sided model, devoid of fitting parameters, demonstrates quantitative agreement with experimental data and confirms that experimentally observed instabilities are driven by thermocapillary stress, and not by the gas convection. By creating creating a numerical infrared image, we can conclude with certitude that the experimentally observed thermo-convective instabilities in evaporating sessile drops of volatile liquids, which in infrared spectrum look similar to hydrothermal waves (HTWs), are actually nothing else than unsteady Bnard-Marangoni instability.
\end{abstract}

PACS numbers: 68.03.Fg, 07.05.Tp, 47.55.pf, 47.20.Ma

Evaporating sessile drops of various liquids are widely encountered in nature and have a variety of industrial and biomedical applications: heat exchangers [? ], nanoparticles deposition (coffee-ring effect) [? ], spraying of herbicides and pesticides on hydrophobic leaves [? ], inkjet printing [? ? ], blood analysis [? ? ? ]. As a result, evaporating sessile drops constitute an interest for both academic community and industry. This problem is rich with numerous physical phenomena: diffusive and convective vapour transport, kinetics of vapour molecules transfer across liquid-gas interface (Hertz-Knudsen-Langmuir equation), evaporative interface cooling, radiative heat transfer, Marangoni flows and instabilities, particles sedimentation and deposition, adsorption of chemicals on interfaces, adsorbed precursor films, contact line instabilities and pinning/depinning processes. Many of these phenomena can be visualized with the aid of modern optical instruments. For example, field of vapour concentration around the droplet can be observed with the aid of digital holographic interferometry [? ]. Observations in infrared (IR) spectrum give visual information about thermal processes in a droplet [? ? ? ? ? ? ], and allow estimating temperature field on its surface [? ? ? ? ]. Among all observable phenomena, a particular interest has been given to the one distinctive type of spontaneously developed thermo-capillary instabilities, so called hydrothermal waves (HTWs). Conventional HTWs are observed in thin liquid layers whose surface is subject to a lateral temperature gradient [? ? ? ? ? ]. In sessile droplets, however, HTWs are driven by evaporation, which generates these temperature gradients naturally. In droplets they have been observed, for the first time, in IR spectrum in volatile liquids (methanol, ethanol, FC-
72) on heated substrates by Sefiane et al. [? ] and later by few other researchers [? ? ? ? ]. According to Smith and Davis [? ? ] (see also [? ] and p. 115 in [? ]), HTWs are distinguished from other thermo-capillary instabilities by the following attributes: they appear only as a secondary unsteady thermo-convective instability in a basic shear flow (primary thermo-capillary flow) directed along the longitudinal temperature gradient at the liquid surface; the mechanism of HTWs propagation does not require any deflection of the free surface of the liquid layer.

It is still not clear what exactly is observed in sessile droplets: are we observing hydrothermal waves or unsteady Benard-Marangoni (BM) instabilities, or a combination of both? In order to answer this question, it is necessary to understand underlying hydrodynamics and heat transfer, identify major sources of IR radiation contributing to the experimentally observed IR image. In the present work, we study this problem numerically using a one-sided model solving 3D unsteady hydrodynamic and heat transfer equations in a sessile drop of ethanol on a heated substrate. We assume a pinned contact line and a spherical-cap shape of the liquid-gas interface. The computed temperature field is used for the numerical reconstruction of the top-view IR image of a semitransparent (in IR spectrum) droplet, which is then validated against the experimental IR image. The presented numerical model gives the idea of internal 3D flow structure in the droplet, reveals the driving mechanism and energy sources of the observed thermo-convective instability, and thus clarifies its nature. For our calculations, we will use a Cartesian system of coordinates (x, y, z) with the origin at the geometrical center of the droplet- 
substrate contact area, and with z-axis directed perpendicular to the liquid-solid interface and pointing into liquid phase. Let $\hat{\mathbf{x}}, \hat{\mathbf{y}}$ and $\hat{\mathbf{z}}$ be the constant unit vectors in direction of $x, y$ and $z$ axes, respectively. Earlier [? ] we have presented a semi-analytical formula predicting the mass flow rate, $J$ (in $\mathrm{kg} / \mathrm{s}$ ), of unsteady diffusion-limited evaporation for a non-isothermal pinned sessile droplet, taking into account Stefan flow in the gas:

$$
\begin{aligned}
J^{\text {Stefan }} & \left(L, T_{a v}, \theta(t), t\right)=2 \pi D_{\text {eff }}^{*} L F(\theta(t)) \\
& \times\left(1+\frac{L F(\theta(t))}{\sqrt{\pi D_{e f f}^{*} t}}\right) \rho_{g}^{*} \ln \left(\frac{\rho_{g}^{*}-\rho_{v, \infty}}{\rho_{g}^{*}-\rho_{v, s a t}^{*}}\right),
\end{aligned}
$$

where

$$
\begin{aligned}
& D_{\text {eff }}^{*}=A \sqrt{T_{a v} T_{\infty}}\left(\sqrt{T_{a v}}+\sqrt{T_{\infty}}\right) / 2 \\
& A=\left(D p_{g}\right)_{r e f} /\left(p_{g} T_{r e f}^{3 / 2}\right) \\
& T_{a v}=\frac{1}{\Gamma} \int_{\Gamma} T d \Gamma, \\
& \rho_{g}^{*}=p_{g} M_{g}^{*} /\left(R T_{a v}\right), \\
& F(\theta)=\left\{\begin{array}{c}
\left(0.6366 \theta+0.09591 \theta^{2}-0.06144 \theta^{3}\right) / \sin \theta \\
0 \leq \theta<\pi / 18 \\
\left(0.00008957+0.6333 \theta+0.116 \theta^{2}\right.
\end{array}\right. \\
& \rho_{v, \text { sat }}(T)=\frac{p_{v, \text { sat }}^{\text {ref }} M_{v}}{R T} \exp \left[-\frac{\Lambda^{*}}{R}\left(\frac{1}{T}-\frac{1}{T_{\text {sat }}^{r e f}}\right)\right] \\
& \rho_{v, \text { sat }}^{*}=\rho_{v, \text { sat }}\left(T_{a v}\right),
\end{aligned}
$$

$L$ is contact radius, $T_{a v}$ and $T_{\infty}$ are correspondingly average temperature of the liquid-gas interface $\Gamma$ (see Eq. (4)) and the ambient one, $t$ is time, $\theta(t)$ is timedependent contact angle, $F(\theta)$ is function of contact angle, derived by Picknett and Bexon [? ] and equivalent to the one derived later by Popov [? ], asterisk stands for a dependence on $T_{a v}: D_{e f f}^{*}$ is effective diffusion coefficient for vapour in ambient gas, $p_{g}$ is ambient gas pressure, $\left(D p_{g}\right)_{r e f}=1.337 \mathrm{~Pa} \cdot \mathrm{m}^{2} / \mathrm{s}$ at $T_{r e f}=298 \mathrm{~K}$ for ethanol vapour in air [? ], $\rho_{g}^{*}$ (see Eq. (5)) and $M_{g}^{*}=$ $M_{a i r}\left(1-X_{v}^{*}\right)+M_{v} X_{v}^{*}$ are correspondingly density and molar mass of the gas at $\Gamma$, where $M_{a i r}$ and $M_{v}$ are molar masses of correspondingly air and vapour $(0.046$ $\mathrm{kg} / \mathrm{mol}$ for ethanol) and $X_{v}^{*}=\rho_{v, s a t}^{*} R T_{a v} /\left(M_{v} p_{g}\right)$ is vapour molar fraction, $\rho_{v, s a t}(T)$ is local saturated vapour density at $\Gamma$ (Clausius-Clapeyron equation, see Eq. (7)), $R$ is the universal gas constant, $\Lambda^{*}=c_{1}\left(1-T_{a v} / T_{c}\right)^{c_{2}}$ is latent heat of vaporization with $c_{1}=55789 \mathrm{~J} / \mathrm{mol}$, $c_{2}=0.31245$ and $T_{c}=514 \mathrm{~K}$ for ethanol, $p_{v \text {,sat }}^{r e f}=13838$ $\mathrm{Pa}$ at $T_{\text {sat }}^{\text {ref }}=308.15 \mathrm{~K}, \rho_{v, \infty}$ is vapour density in the ambient gas far away from the droplet. Equation (1) has been used in a two-parametric approximation, $j_{\text {approx }}$, for a non-isothermal vapour flux distribution along the droplet surface (see [?]):

$$
j_{\text {approx }}=j_{i}^{*}(r, \theta)\left[1+B\left(T-T_{a v}\right)\right]+\Delta h \nabla_{\Gamma} \cdot \mathbf{j}_{\tau},
$$

where

$$
\begin{aligned}
& r=\sqrt{x^{2}+y^{2}} \\
& \mathbf{j}_{\tau}=-D(T) \nabla_{\Gamma} \rho_{v, s a t}(T)+\rho_{v, s a t}(T) \mathbf{u}_{\tau},
\end{aligned}
$$

$B$ and $\Delta h$ are two parameters, $\boldsymbol{\nabla}=\hat{\mathbf{x}} \frac{\partial}{\partial x}+\hat{\mathbf{y}} \frac{\partial}{\partial y}+\hat{\mathbf{z}} \frac{\partial}{\partial z}$ is the nabla-operator, $\boldsymbol{\nabla}_{\Gamma}=(\mathbf{I}-\mathbf{n n}) \cdot \boldsymbol{\nabla}$, where $\mathbf{I}$ is the identity tensor and $\mathbf{n}$ is unit normal vector at the liquidgas interface pointing into gas phase, $\mathbf{j}_{\tau}$ is tangential component of the vapour flux (see Eq. (11)), $D(T)=A T^{3 / 2}$ is local diffusion coefficient, $\mathbf{u}_{\tau}=(\mathbf{I}-\mathbf{n n}) \cdot \mathbf{u}$ is tangential component of the velocity vector, $\rho_{v, s a t}(T)$ is local saturated vapour density, $\boldsymbol{\nabla}_{\Gamma} \cdot \mathbf{j}_{\tau}$ is a surficial divergence (see Eq. (23)) of the surficial vector $\mathbf{j}_{\tau}$, and finally $j_{i}^{*}(r, \theta)$ is an isothermal version of the vapour flux distribution along the droplet surface:

$$
\begin{aligned}
& j_{i}^{*}(r, \theta)=j_{0}^{*} \chi^{-\lambda}(1-\omega) \\
& \text { for } 0 \leq \theta \leq \pi / 2 \text { and } 0 \leq r<L
\end{aligned}
$$

where

$$
\begin{aligned}
& j_{0}^{*}=\frac{J^{\text {Stefan }}\left(L, T_{a v}, \theta(t), t\right) G(\theta)}{2 \pi L^{2} F(\theta)}, \\
& \begin{aligned}
G(\theta)= & 0.008348 \theta^{4}-0.1026 \theta^{3} \\
& +0.001815 \theta^{2}+0.4491 \theta+0.6368,
\end{aligned} \\
& \begin{aligned}
\chi= & -(r / L)^{2}, \\
\lambda= & (\pi-2 \theta) /(2 \pi-2 \theta), \\
\begin{array}{c}
\omega \\
\omega
\end{array} & \left\{\begin{array}{l}
\left.H-\sqrt{4+5\left(1-\theta / \theta_{H E}\right)^{2}}\right], \\
-E+\sqrt{\lambda_{E}^{2} E^{2}+\left(1-\lambda_{E}^{2}\right)(H+E)^{2}},
\end{array}\right. \\
\lambda_{E}= & \left(2 \theta-2 \theta_{H E}\right) /\left(\pi-2 \theta_{H E}\right), \\
\theta_{H E}= & 0.7864 H+0.9103, \\
E= & -2.679 H+0.7265, \\
H= & 0.26\left(1-\chi^{0.7}\right) .
\end{aligned}
\end{aligned}
$$

Defining liquid-gas interface $\Gamma: z=f(x, y)$, we can represent $\mathbf{j}_{\tau}$ as a function $\mathbf{v}$ of only two coordinates:

$$
\mathbf{j}_{\tau}(x, y, z)=\mathbf{j}_{\tau}(x, y, f(x, y)) \equiv \mathbf{v}(x, y) .
$$

Then, provided that $n_{z} \neq 0$, term $\nabla_{\Gamma} \cdot \mathbf{j}_{\tau}$ in Eq. (9) can be expressed as:

$$
\nabla_{\Gamma} \cdot \mathbf{j}_{\tau}=\mathbf{a} \cdot(\mathbf{v})_{x}^{\prime}+\mathbf{b} \cdot(\mathbf{v})_{y}^{\prime}
$$

where symbols $(\cdot)_{x}^{\prime}$ and $(\cdot)_{y}^{\prime}$ denote partial derivatives with respect to $x$ and $y$, and vectors $\mathbf{a}$ and $\mathbf{b}$ are given as follows:

$$
\begin{aligned}
& \mathbf{a}=(\mathbf{I}-\mathbf{n n}) \cdot \hat{\mathbf{x}}=\left(\begin{array}{lll}
1-n_{x}^{2}, & -n_{x} n_{y}, & -n_{x} n_{z}
\end{array}\right), \\
& \mathbf{b}=(\mathbf{I}-\mathbf{n n}) \cdot \hat{\mathbf{y}}=\left(\begin{array}{lll}
-n_{y} n_{x}, & 1-n_{y}^{2}, & -n_{y} n_{z}
\end{array}\right),
\end{aligned}
$$


with $n_{x}=-f_{x}^{\prime} n_{z}, n_{y}=-f_{y}^{\prime} n_{z}$ and $n_{z}=\frac{1}{\sqrt{1+\left(f_{x}^{\prime}\right)^{2}+\left(f_{y}^{\prime}\right)^{2}}}$ being the components of the normal vector $\mathbf{n}$. Expression (23) is ready to be implemented in the program code. We use Eq. (9) in our numerical model as a boundary condition for the local normal vapour flux, $j$, at the liquid-gas interface: $j=j_{\text {approx }}$. All input data, which are required for the computation of $j_{\text {approx }}$, such as $T_{a v}, \theta(t)$, $\mathbf{j}_{\tau}$, are directly available from the numerical model in the course of computation. Our one-sided model solves Navier-Stokes, continuity and heat transfer equations in the droplet bulk:

$$
\begin{aligned}
& \rho\left(\left.\frac{\partial \mathbf{u}}{\partial t}\right|_{m}+[(\mathbf{u}-\mathbf{w}) \cdot \boldsymbol{\nabla}] \mathbf{u}\right)=\boldsymbol{\nabla} \cdot \boldsymbol{\sigma} \\
& \boldsymbol{\nabla} \cdot \mathbf{u}=0 \\
& \rho c_{p}\left(\left.\frac{\partial T}{\partial t}\right|_{m}+(\mathbf{u}-\mathbf{w}) \cdot \boldsymbol{\nabla} T\right)=\boldsymbol{\nabla} \cdot(k \boldsymbol{\nabla} T),
\end{aligned}
$$

where $\mathbf{u}$ and $T$ are respectively velocity and temperature fields, w is the mesh velocity, subscript $m$ means that time derivative is taken at a fixed mesh node (fixed mesh coordinates), $\rho, c_{p}$ and $k$ are respectively density, specific heat capacity at constant pressure and thermal conductivity of the droplet phase, $\boldsymbol{\sigma}=-p \mathbf{I}+\boldsymbol{\pi}$ is total stress tensor, $p$ is hydrodynamic pressure and $\boldsymbol{\pi}=\mu\left(\boldsymbol{\nabla} \mathbf{u}+(\boldsymbol{\nabla} \mathbf{u})^{\mathrm{T}}\right)$ is viscous stress tensor. Radiative, conductive and convective heat transfer into gas phase is neglected. Substrate is considered isothermal with constant temperature $T_{s}$. Initial conditions: $\left.T\right|_{t=0}=T_{s}$, $\left.\mathbf{u}\right|_{t=0}=0,\left.p\right|_{t=0}=p_{g}+2 \gamma \sin \theta_{0} / L$, where $\gamma$ is liquid-gas interfacial tension and $\theta_{0}$ is initial contact angle. Boundary conditions: $\left.T\right|_{z=0}=T_{s},\left.\mathbf{u}\right|_{z=0}=0$. Liquid-gas interface $\Gamma$ is considered as a boundary, moving in direction of normal vector $\mathbf{n}$ with velocity $u_{\Gamma}$. Provided that local evaporation rate, $j$, is known (see Eq. (9)), we can define the following boundary conditions at $\Gamma$ :

$$
\begin{aligned}
& -k \boldsymbol{\nabla} T \cdot \mathbf{n}=j \Lambda^{*} / M_{v}, \\
& \rho\left(\mathbf{u} \cdot \mathbf{n}-u_{\Gamma}\right)=j \\
& \boldsymbol{\sigma} \cdot \mathbf{n}=-\gamma(\boldsymbol{\nabla} \cdot \mathbf{n})_{\Gamma} \mathbf{n}+\frac{d \gamma}{d T} \nabla_{\Gamma} T
\end{aligned}
$$

where $(\boldsymbol{\nabla} \cdot \mathbf{n})_{\Gamma}$ is the divergence of vector $\mathbf{n}$ at $\Gamma$, that is curvature of $\Gamma$, which is equal to $2 \sin \theta / L$. Expression for $u_{\Gamma}$ is derived based on total evaporative mass flux $J=\int_{\Gamma} j d \Gamma$ and two assumptions: pinned contact line ( $L=$ const) and spherical-cap shape of the droplet in the course of evaporation:

$$
u_{\Gamma}=-\frac{J}{\pi \rho L^{3}} \frac{(1+\cos \theta)^{2}}{\sin \theta} z .
$$

In order to compare our numerical model with real experimental data, we have selected one particular experiment from the series of our parabolic-flight experiments [? ]. In this experiment a sessile drop of ethanol with pinned contact line evaporated in microgravity conditions from

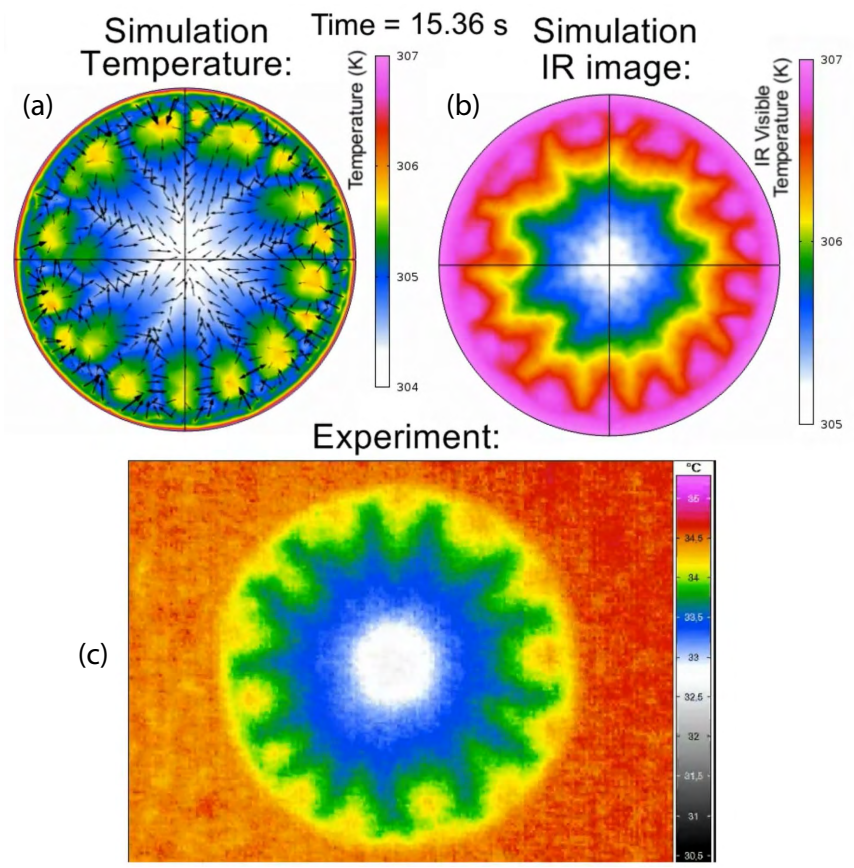

FIG. 1. Snapshot of comparison between experimental and numerical infrared images at $\mathrm{t}=15.36 \mathrm{~s}$ : a) top left figure evidence the numerical droplet surface temperature. b) top right figure evidence the numerical droplet total radiative temperature. c) bottom central figure show the experimental infrared image obtained for an ethanol droplet under evaporation for the same conditions. Multimedia view

a thin heated substrate with actively imposed constant temperature $T_{s}$. The IR camera "VarioCam ${ }^{\circledR}$ hr head" recorded droplet's top view in the Mid-Wavelength IR range (MW: 7.5-14 $\mu \mathrm{m}$ ) to capture thermo-convective instabilities (Fig. 1c). Based on the selected experiment, we have used the following input data for our numerical model: $p_{g}=792 \operatorname{mbar}, T_{s}=307.05 \mathrm{~K}, T_{\infty}=297.55$ $\mathrm{K}, L=2.95 \mathrm{~mm}, \theta_{0}=29.2^{\circ}, \rho_{v, \infty}=0, \rho=772.24$ $\mathrm{kg} / \mathrm{m}^{3}, \mu=1.095 \mathrm{mPa} \cdot \mathrm{s}, k=0.14 \mathrm{~W} /(\mathrm{m} \cdot \mathrm{K}), c_{p}=2602.3$ $\mathrm{J} /(\mathrm{kg} \cdot \mathrm{K}), \gamma=20.62 \mathrm{mN} / \mathrm{m}, \frac{d \gamma}{d T}=-82 \mu \mathrm{N} /(\mathrm{m} \cdot \mathrm{K}) . \mathrm{Pa}-$ rameters $B=0.33 \mathrm{~K}^{-1}$ and $\Delta h=0.019 \mathrm{~mm}$ (see Eq. (9)) were obtained by fitting local evaporation rate, $j$, computed with our previously developed non-isothermal two-sided axisymmetric numerical model [? ] which also used the above mentioned input data.

In the present numerical study we use a commercial software COMSOL Multiphysics ${ }^{\circledR}$. All equations are discretized with the finite element method (FEM) using second order shape functions. Generated computational mesh contains 16547 tetrahedral elements, which results in 187050 degrees of freedom. Computation ran about 9 days on a cluster with 48 cores. Below we present computer simulation results for our unsteady one-sided 3D numerical model. Computation starts with the initial condition of uniform temperature field in the droplet 


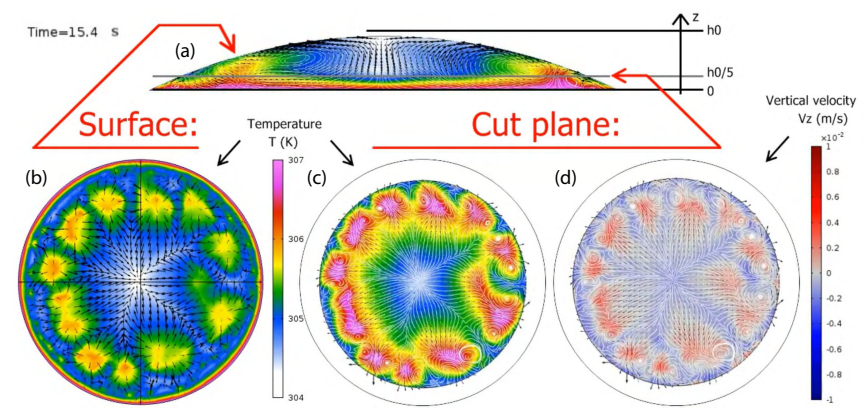

FIG. 2. Snapshot of flow motion inside the droplet under evaporation from the numerical simulation at $\mathrm{t}=15.40 \mathrm{~s}$ : a) top figure showing the thermal plume formation inside the droplet. b) bottom left figure showing the top surface temperature. c) central figure showing in a cut plane the liquid temperature and d) on the right view the vertical velocity component. Multimedia view

bulk. Due to consumption of latent heat by the theoretically imposed evaporation at the liquid-gas interface, droplet surface cools down. At the same time temperature near the contact line is maintained high due to heat conduction from the substrate through a thin layer of ethanol. This creates a vertical temperature gradient in the droplet bulk and a horizontal one on the droplet surface near the contact line. These temperature gradients promote the development of thermocapillary Benard-Marangoni instability. After a period of about 15.4 seconds, when intensive transitional phenomena finished, one can observe a dynamic multicellular thermo-convective pattern: Fig. 2 shows the temperature (color) and velocity (arrows) fields at the droplet surface (b) and in the vertical cut plane (a) after $15.40 \mathrm{sec}-$ onds of the evaporation process which represents about $85 \%$ of the total time of evaporation. The dynamics of this instability well resembles the experimentally observed one (a video link is available in the caption of Fig. 1). In order to get a better visual comparison of this numerical result with the experiment, we did a simplified computation of the IR image based on the computed $3 \mathrm{D}$ temperature field. We took into account the semitransparency of liquid ethanol in the Mid-Wavelength (MW) bandwidth: according to the spectrophotometer measurements by Brutin et al. [? ] ethanol's absorption coefficient in the MW range is $a=1.85 \mathrm{~mm}^{-1}$. Using this value, one can compute the intensity of IR radiation arriving to the camera installed above the droplet, which then can be converted into an equivalent blackbody temperature, $T_{I R, \text { num }}(x, y)$ (subscript num stands for "numerical"). This computation is done by summing the IR radiation coming from the substrate surface (assumed to be a gray body) with the integral of IR radiation sources distributed across the thickness of a semi- transparent droplet:

$$
\begin{aligned}
& T_{I R, \text { num }}^{4}(x, y)=\varepsilon T_{s}^{4} e^{-a f(x, y)}+ \\
& \int_{0}^{f(x, y)} T^{4}(x, y, z) a e^{-a(f(x, y)-z)} d z,
\end{aligned}
$$

where $f(x, y)$ represents the local droplet thickness. Emissivity of a bare substrate surface, $\varepsilon=$ $T_{I R, \exp }^{4} / T_{s, \text { exp }}^{4}$, is estimated by correlating the experimental equivalent blackbody temperature $\left(T_{I R, \exp }(x, y)\right.$ from IR camera) with corresponding real temperature, $T_{s, e x p}$, obtained through thermocouple measurements.

Numerical model does not confirm the existence of any base shear flow in the droplet phase, which indicates that this is not HTWs. Instead, it possesses the features of Benard-Marangoni instability: vertical upward liquid flow under the local temperature maxima, and downward flow under the local temperature minima. Thus, we conclude that the experimentally observed thermoconvective pattern is nothing else than unsteady BenardMarangoni instability.

Finally, let us discuss the dynamics of the observed pattern. The 3D instabilities develop in several stages. In the first one, an unsteady axisymetric base flow sets up in a torus roll adjacent to the triple line. It rotates upwards along the interface as it is driven by thermocapillary forces and superimposes to Stefan flow in the droplet. In a second stage this torus roll is destabilised and brakes into BM cells resulting in a fully 3D unsteady pattern that populates the whole droplet. Then in a third stage the BM cells concentrate in the neighbouring of the triple line where thermocapillary forces remain intense whereas they continuously weaken in the upper central region. The unsteady pattern that arises from this point results from the fact that BM cells have an intrinsic wavelength that is roughly twice the liquid thickness $[?](\lambda$ $\sim 2 \mathrm{f}(\mathrm{r}))$. So the number of BM cells that matches the circumference is $N(t) \sim \frac{2 \pi(L-f(r))}{2 f(r)}$. But, as evaporation proceeds in the pinned mode, the droplet thickness decreases and $\mathrm{N}(\mathrm{t})$ increases leading to a continuous azimuthal movement in order to dynamically equidistribute cells along the droplet perimeter. This last dynamics has somehow been erroneously attributed to HTWs, but our comparison of experiments and simulation confirms the nature of these instabilities as BM.

In this letter we have reported our 3D one-sided numerical model of an evaporating sessile droplet of ethanol on a heated substrate. The model does not use any fitting parameters and demonstrates a good qualitative and quantitative comparison with the experimental data. Based on the computed temperature and flow fields we conclude that the experimentally observed thermoconvective pattern in the droplet is an unsteady BenardMarangoni instability and is different from hydrothermal waves.

We would like to acknowledge the financial support of 
the "Centre National d'Études Spatiales" research grant for post-doctorates and the European Space Agency. This work has been carried out in the framework of Labex
MEC (ANR-10-LABX-0092) and A*MIDEX (ANR-11IDEX-0001-02) projects, funded by the Investissements d'Avenir French Government program managed by the French National Research Agency. 